\title{
Splitting and Merging Algorithm Based on Local Statistics of Sub-Regions in Document Image
}

\author{
Kiran Thapaliya, Il-Cheol Park, and Goo-Rak Kwon, Member, KIMICS
}

\begin{abstract}
This paper presents splitting and merging algorithm based on adaptive thresholding. The algorithm first divides the image into blocks, and then compares each block using the calculated thresholding value. The blocks which are same are merged using the certain threshold value and different blocks are split unless it satisfies the threshold value. When the block has been merged, maximum and minimum block sizes are determined then the average block size is determined. After the average block size is determined the average intensity and standard deviation of average block is calculated. The process of thresholding is applied to binarize the image. Finally, the experimental results show that the proposed method distinguishes clearly the background with text in the document image.
\end{abstract}

Index Terms - standard deviation, image enhancement, split and merge, mean.

\section{INTRODUCTION}

THE most important thing that we need is the information. So information should be easily readable. Basically, historical text documents are difficult to read so one of the basic techniques for the extraction of the text is thresholding. It is the conversion of gray level or color image into the black or white image or conversion of the pixel which is in the range of $0-256$ into 0 or 1 form. As the time passes we have different documents which have to be preserved for the future references. But the documents which are preserved may be distorted by various means like light illumination, noise, dust and many other factors. So the historical documents are really difficult to read and identify the text written on it. Various algorithms have been proposed by different authors so that the historical documents can be read effectively. If the background is uniform, it can read the text. However it is difficult to read the text when the document is not uniform. Niblack [2] algorithm is based on the local statistics of the image and works well but fails when the background of the image has light texture. Niblack [2] used the following equation to calculate the threshold

\footnotetext{
Manuscript received July 6, 2011; revised August 9, 2011; accepted August 18, 2011.

Kiran Thapaliya, Il-Cheol Park, and Goo-Rak Kwon are with the Department of Information and Communication Engineering, Chosun university, Gwangju, 501-759, Korea (Email: grkwon@chosun.ac.kr)
}

value $\mathrm{T}(\mathrm{x}, \mathrm{y})$ at pixel $(\mathrm{x}, \mathrm{y})$ using the following equation:

$$
T(x, y)=m(x, y)+k . s(x, y),
$$

Where $\mathrm{m}(\mathrm{x}, \mathrm{y})$ and $\mathrm{s}(\mathrm{x}, \mathrm{y})$ are the mean and standard deviation of local area. Sauvola [3] proposed a new method which is the modified form of the Niblack's method by adding the hypothesis in the background and text in the image. It uses the following formula for the threshold.

$$
T(x, y)=m(x, y)+1-k\left(1-\frac{s(x, y)}{R}\right),
$$

Where $\mathrm{m}(\mathrm{x}, \mathrm{y})$ and $\mathrm{s}(\mathrm{x}, \mathrm{y})$ are the mean and standard deviation of the local area and $\mathrm{R}$ is the dynamics of the standard deviation fixed to 128 and $\mathrm{k}$ is fixed value usually set to 0.5 .

Gatos [5] proposed the binarization technique for the poor document and he has used Sauvola [3] technique to determine the foreground of an object. Gatos [5] used the following formula:

$$
I(x, y)=\mu+\left(\left(\sigma^{2-v^{2}}\right)(f(x, y)-\mu)\right) / \sigma^{2},
$$

Where $f(x, y)$ is the gray level source image, $\mu$ is the local mean, $\sigma 2$ is the variance at $3 \times 3$ neighborhood around each pixel and $\mathrm{v} 2$ is the average of all estimated variance for each pixel in the neighborhood. These all method works well for the poor quality document image. Since thresholding value of each pixel should be determined. The computational complexity is high.

This paper presents a new algorithm based on the splitting and merging of text image using the local statistics of the image.

\section{PROPOSED ALGORITHM}

In Fig. 1, splitting and merging is such a recursive approach which takes the image as input and divides the image into four different parts. Then the image consists of four different sub-images. The further split takes place in the sub-images if the homogeneity is not same. It applies the same operation to the non-homogenous sub-image region. 
In this section, the algorithm for enhancement of the text document is proposed which divides the whole image into small blocks based on the local statistics of the pixel. The basic process of splitting is shown in Fig.1 (a). The given image is split into four parts and the sub-region is further sub-divided into different sub-regions unless the some thresholding criteria is satisfied. Fig.1 (b) shows the hierarchical splitting of the Fig.1 (a).

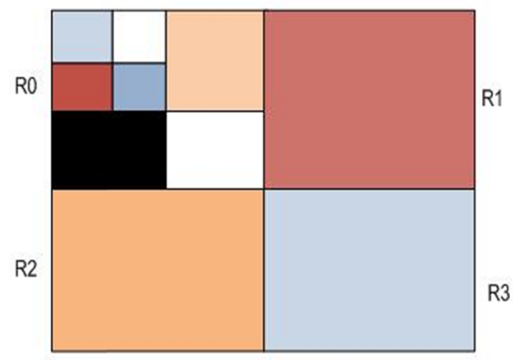

(a)

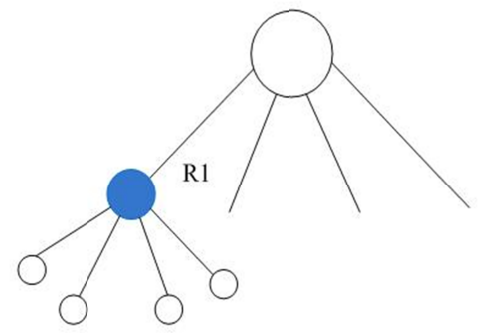

(b)

Fig. 1. Normal Split-Merge.

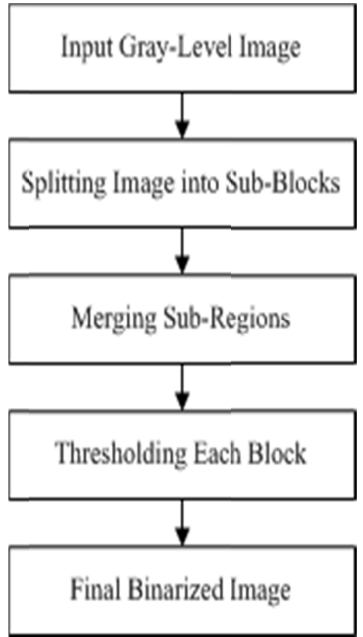

Fig. 2. Procedure of the proposed algorithm.

\section{A. Calculating the basic threshold parameter}

For the splitting of the image into different sub-region, some thresholding value should be determined. Therefore the input image is taken and its mean and standard deviation is determined. The determined mean and standard deviation is the global mean and standard deviation of the image. The basic thresholding value is determined by using the following formula.

$$
T=a * m+b * s t,
$$

Where $\mathrm{m}$ and st are the mean and standard deviation of the image, respectively. $\mathrm{a}$ and $\mathrm{b}$ are any constants whose value is determine experimentally. Using this thresholding value $T$, the image is split into different sub-regions.

\section{B. Splitting}

Splitting operation splits the image into four different parts. It takes the thresholding value $T$ which is calculated in Equ. (4) and splits the image into four different subimages. The different sub-regions are checked with the thresholding value $T$. If the sub-regions satisfy the threshodling value $T$, they are not further split into subregions else they are split into more sub-regions unless they satisfy the threshodling criteria $T$.

\section{Merging}

Merging is the process of adding the sub-regions which has been split into different sub-regions. The sub-regions that are similar are merged with one another and subregion that are not similar are left out. Therefore, the merging of the different sub-regions is done by following the two different steps.

1) Calculating the average block size and average intensity of block

After the splitting of image into different sub-region, the average block size of sub-region is calculated. Then the average mean and the standard deviations value of the average block is calculated.

Equ. (5) calculates the average block size. Equ. (6) calculates mean $(\mu)$ of an average block. Equ. (7) calculates standard deviation $(\sigma)$ of average block. The thresholding value is calculated by the addition of the mean and standard deviation of average block which is shown in Equ. (8).

$$
\begin{aligned}
& \operatorname{avg}(\text { block })=\frac{\text { numbers of pixel in each block }}{\text { number of blocks }}, \\
& \mu(\text { block })=\sigma(\text { avg }(\text { block })) \\
& \sigma(\text { block })=\sigma(\operatorname{avg}(\text { block })) \\
& T=\mu+\sigma,
\end{aligned}
$$

Where $\mu$ and $\sigma$ are the mean and standard deviation of the average block.

\section{2) Thresholding each block}

As it is known that the standard deviation of the image gives the diversity of the pixel in an image and the mean of the image gives the intensity of the image, therefore the 
final thresholding of the image is explained below.

Then mean and standard deviation of the each subregion is calculated. If the standard deviation of each sub-region is less than the predefined standard deviation $(\sigma \leq \sigma T)$ then the sub-region may contain either the background pixel or foreground pixel. If the mean of the each region is greater than the predefined mean value $(\mu \geq \mu T)$ then the region contains only the brighter pixel. The final binarization of each sub region is done by using the Equ. [9]. If the sub-region has the value greater than $T$ obtained from the Equ. [9] then the region is merged and labeled as object else it is labeled as background. The value of $\sigma_{T}$ and $\mu_{T}$ are determined experimentally and found as 7 and 9 respectively for the document images.

\section{SIMULATION AND RESULTS}

The proposed algorithm is verified by testing in different kinds of document images. The simulation is performed on MATLAB 9.0. Documents are taken from the newspaper and other different sources.

In Fig.3 (a) shows the original input image. This image contains a lot of background noises which makes difficult for people to read the object clearly. The input image is tested using different algorithm. Fig.3 (b), Fig.3 (c), and Fig.3 (d), shows the output image obtained using Otsu method, Niblack method and Sauvola method.Fig.3 (e) shows the output image obtained using the proposed method. The original image when passed through the proposed algorithm gives the better experimental result as compared with other three different algorithms. The text is not clear and noises are retained with three different methods but with the proposed algorithm, the text is clear and noises are removed from the image which makes the text clear and easier to read. Fig. 4 also shows the comparison of experimental results between proposed algorithm and other three methods. As it clear from the experimental results that proposed algorithm yields better results than the other three different methods. Fig.5 (a), Fig.6 (a), Fig.7 (a), and Fig.8 (a) are the original text document image and Fig.5 (b), Fig.6 (b), Fig.7 (b), and Fig.8 (b) are the output images respectively, obtained experimentally using proposed algorithm. Therefore, the proposed algorithm was applied to the different types of image and the result achieved was convenient. Thus, the experimental results when applied to different kinds of text documents yields better result than the other three different methods and proves that proposed algorithm is better and gives better result in comparison to other methods.
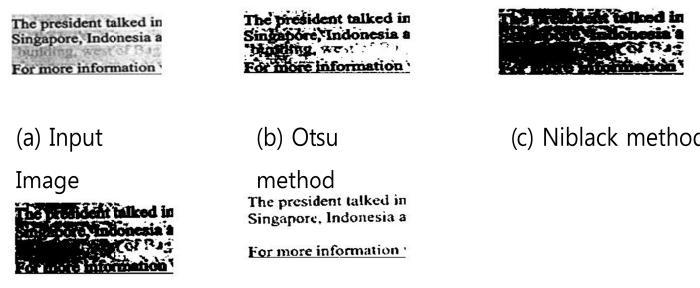

(b) Otsu

(c) Niblack method

(d) Sauvola method

method

The president talked in
Singapore, Indonesia a

For more information

(e) Proposed

method

Fig. 3. Simulation results obtained using different methods and proposed method.

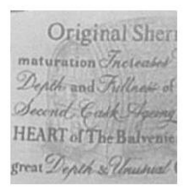

(a) Original Image

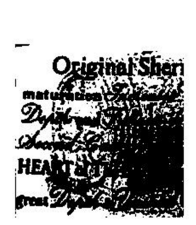

(d) Sauvola method

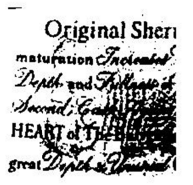

(b) Otsu method

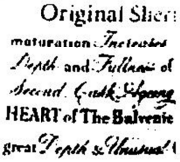

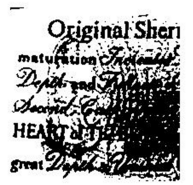

(c) Niblack method
Fig. 4. Simulation results obtained using different methods and proposed method.

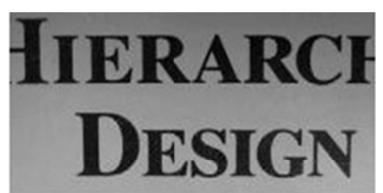

(a) Original Image

\section{IIERARCF DESIGN}

(b) Binarized Image
Fig. 5. Simulation result of text using proposed method.

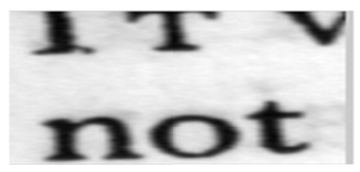

(a) Original Image

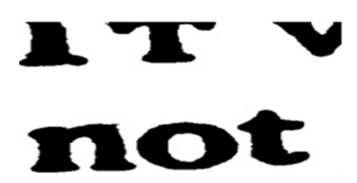

(b) Binarized Image
Fig. 6. Simulation result of text using proposed method. 


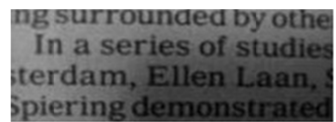

(a) Original Image ng surrobiacien iny othet In a series of studies chrdam, Ellen Latans ipir'ting demonstrated

(b) Binarized Image
Fig. 7. Simulation result of text using proposed method.

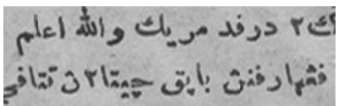

(a) Original Image

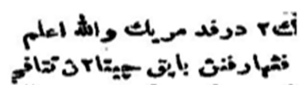

(b) Binarized Image
Fig. 8. Simulation result of text using proposed method.

\section{CONCLUSIONS}

In this paper, we have presented the algorithm for the thresholding of the text document. The algorithm uses the splitting and merging techniques based on the local statistics of the sub-regions. The mean and the standard deviation of the sub-region is calculated and compared with the predetermined value and finally the binarized image is achieved by using the thresholding value $T$. Since the sub-regions are merged instead of each pixel the complexity is also reduced. Finally, the proposed algorithm works for the text document with the different background.

\section{REFERENCES}

[1] N. Otsu, "A threshold selection method from grey level histogram," IEEE Trans. Syst. Man Cybern., Vol. 9, No. 1, pp. 62-66, 1979.

[2] W. Niblack, "An introduction to digital image processing," Prentice Hall, 1986.

[3] J.Sauvola and M.pietikainen, "Adaptive document image binarization," Pattern Recognition, vol. 33, pp. 225-236, 2000.

[4] Abutaleb, A.S. "Automatic thresholding of gray-level pictures using two-dimensional entropy," Comput. Vis. Graph Image Process, 47, pp. 22-32, 1989

[5] B. Gatos, I. Pratikakis, and S. J. Perantonis, "Adaptive degraded document image binarization," Pattern Recogntion, vol. 39, pp. 317 327,2006

[6] Leedham, G., S. Varma, A. Patankar, and V. Govindaraju "Separating Text and Background in Degraded Document Images," Proceedings Eighth International Workshop on Frontiers of Handwriting Recognition, pp. 244-249, Sept. 2002.

[7] P. K. Sahoo, S. Soltani, A. K. C. Wong, and Y. C. Chen, "A survey of thresholding techniques," Computer Vision, Graphics and Image Processing, vol. 41, pp. 233-260, 1988.

[8] S. D. Yanowitz and A. M. Bruckstein, "A new method for image segmentation," Computer Vision, Graphics and Image, 46, (1), pp. 82$95,1989$.

[9] Ali El Zaart, Ali Al-Mejrad, and Ali Saad, "Segmentation of mammography images for breast cancer detection," In the Proceedings of the Kuala Lumpur International Conference on Biomedical Engineering 2004, pp. 225-228. Sept. 2004.

[10] Da-Wen Sun and Cheng-Jin Du "Segmentation of complex food images by stick growing and merging algorithm," Journal of visual communication and image representation, vol. 18, pp. 119-129, 2007.

[11] M. Marina, S. Karl, and K. Hermann, "Edge and region based segmentation technique for the extraction of large, man-made objects in high resolution satellite imagery," Pattern Recognition, 37(8), pp. 1619628, 2004.

[12] Yaowen Zhan, Weiqiang Wang, and Wen Gao "A Robust split - merge text segmentation approach for images," in 8th International Conference on Pattern Recognition (ICPR'06), 2006.

[13] X. Wu, "Adaptive split and merge segmentation based on piecewise least-square approximation," IEEE Trans. Patt. Anal. Machine Intell, vol. 255, No. 8, pp. 808-815, Aug. 1993.
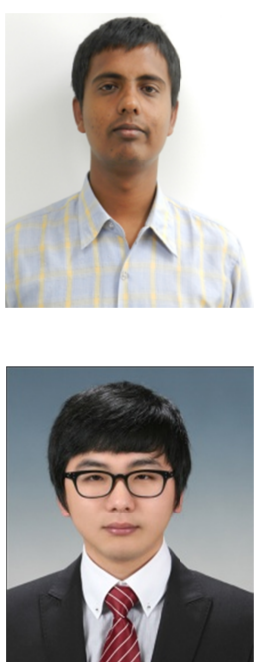

Il-Cheol Park received his B.S. degree in information and communication engineering from the University of Chosun, Gwangju, South Korea, in 2010. Since March 2010, he has been the M.S. Student in information and communication engineering, University of Chosun, South Korea. His research interests include multimedia image processing, still image codec, video codec.

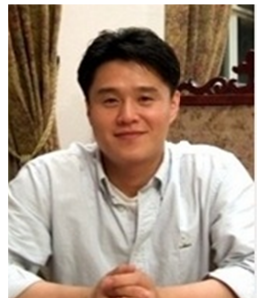

Goo-Rak Kwon received the M.S. degree from SungKyunKwan University, in Electronic Engineering, in 1999. He also received a Ph.D. degree from Korea University in Mechatronics in 2007. In 2008, he joined the Department of Information and Communication Engineering at Chosun University where he is currently an assistant professor. His interest research fields are $\mathrm{A} / \mathrm{V}$ signal processing, multimedia communication, and applications. $\mathrm{He}$ is currently a Member in the IEEE, IEICE, and IS\&T. He has been a Member in the Institute of Electronics Engineers of Korea (IEEK). And also he has served as an Editor for Journal of the Multimedia Society of Korea (KMMS). 